\title{
Individuelle Therapie im Ramadan und mit deutschem Bierbauch
}

\author{
Die Zauberformel von einer individualisierten, auf spezi- \\ fische Patientenbedürfnisse zugeschnittenen Therapie \\ steht seit kurzem auch im diabetologischen Umfeld \\ hoch im Kurs. Bei einer Veranstaltung in Istanbul wurde \\ diese zunächst abstrakte Formulierung gleich mehrfach \\ mit Leben gefüllt.
}

Den spezifischen Bedürfnissen muslimischer Typ-2-Diabetiker hat sich die VECTOR-(Vildagliptin Experience Compared To gliclazide Observed during Ramadan-)Studie angenommen (Hassanein M. Curr Med Resp Opin. 2011;27: 1367-74). In der prospektiv angelegten Beobachtungsstudie konnte gezeigt werden: Eine Behandlung mit zweimal $50 \mathrm{mg}$ Vildagliptin täglich während der Fastenzeit führt im Vergleich zu einem Sulfonylharnstoff ( $80 \mathrm{mg}$ Gliclazid einmal täglich) nicht nur zu einer besseren Stoffwechselkontrolle. Im Unterschied zu dem
Sulfonylharnstoff traten unter der Behandlung mit Vildagliptin (Galvus ${ }^{\circ}$ ) überhaupt keine Hypoglykämien auf, berichtete Prof. Stefano Del Prato, leitender Endokrinologe an der Universität Pisa. Die Unterschiede waren hoch signifikant.

Das für den Abbau von Inkretinen wie GLP-1 verantwortliche Enzym Dipeptidylpeptidase-4 wird nach Darstellung von Prof. Matthias Blüher, Diabetologe an der Universität Leipzig, vor allem im viszeralen Fettgewebe exprimiert und aktiviert. Dies macht DPP-4-Hemmer wie Vildagliptin zu einem besonders geeigne- ten Kandidaten bei Typ-2-Diabetikern mit „deutschem Bierbauch“, trug der Endokrinologe nationalen und pathophysiologischen Gegebenheiten beim Typ-2Diabetes-mellitus gleichermaßen Rechnung.

Im Rahmen der Studie VERIFY (Vildagliptin Efficacy in combination with metfoRmIn For earlY treatment of type 2 diabetes) wird in den kommenden Jahren der Nutzen einer frühzeitigen Kombinationstherapie von Vildagliptin und Metformin untersucht. Aus pathophysiologischem Blickwinkel gibt es für den frühen Einsatz von DPP-4-Hemmern viele Argumente - u.a. das im Raum stehende betazellprotektive Potenzial. Im Klassenvergleich zeichnet sich Vildagliptin durch seine vergleichsweise ausgeprägten Effekte auf den nächtlichen Nüchternblutzucker aus, betonte der Chemiker Edwin Villhauer der bei Novartis Pharmaceuticals in New Jersey/ USA tätig ist und bei der Entwicklung des Wirkstoffs von Anfang an dabei war.

Dr. med. Ludger Riem, Rösrath

${ }^{*}$ Galvus ${ }^{\circledast}$ GOLD 2012: „Reviewing the clinical evidence to individualise patient care" , Istanbul, 15./16. Juni 2012, Veranstalter: Novartis
Viele Blutzuckermessgeräte erfüllen Mindestanforderungen an Messqualität nicht

In zwei Studien hat das Institut für Diabetes Technologie in UIm die Messgenauigkeit von Blutzuckermessgeräten und die Qualität der entsprechenden Teststreifen im Hinblick auf die DIN ISO-Norm untersucht [1, 2]. Diese zeigen, dass nur relativ wenige Systeme zur Blutzuckerselbstmessung die aktuelle und die zukünftige, verschärfte ISO-Norm 15197 erfüllen. Während ca. 80\% der Geräte die derzeitigen Vorgaben schafften, fiel nach der neuen Norm beinahe jedes zweite System durch den Qualitätstest. Auch die Teststreifen wiesen starke Qualitätsunterschiede auf. Bis zu 13\% Abweichung zwischen verschiedenen Chargen eines Herstellers konnten die Forscher nachweisen. Gut abgeschnitten haben die Systeme von Accu-Chek ${ }^{\circledast}$. Sie erfüllen sowohl die aktuellen Anforderungen der ISO Norm als auch die des neuen ISO 15197 Vorschlags. Accu-Chek ${ }^{\circledR}$ Aviva zeigte besonders geringe Abweichungen innerhalb verschiedener Teststreifenchargen.

Die Blutzuckerselbstmessung ist ein wichtiger Bestandteil des Diabetes Selbstmanagements, da die Messwerte ausschlaggebend für die Therapieentscheidungen sind. Anhand der gemessenen Werte wird beispielsweise bestimmt, wie viel Insulin zu einer Mahlzeit verabreicht werden muss. Blutzuckermesssysteme müssen daher zuverlässig exakte Testergebnisse liefern, und das dauerhaft.

1. Freckmann G et. al. J Diabetes Sci Technol. 2012;6(5):1060-75.

2. Baumstark A et. al. J Diabetes Sci Technol. 2012;6(5):1076-86.

Quelle: Roche Diagnostics
Aktuelle Umfrage belegt hohe Zufriedenheit mit MEDIAS 2-Schulungs- und Behandlungs-Programmen

Eine aktuelle Umfrage bei 800 Schwerpunktpraxen [1] zeigt, dass sich MEDIAS 2 BASIS und MEDIAS 2 ICT einer großen Beliebtheit erfreuen. Bei fast allen Befragten, die die beiden Programme kennen, besteht eine hohe Zufriedenheit.

Seit 2001 haben mehr als 6500 Teilnehmer die MEDIAS 2 Seminare besucht - dies sind etwa 3250 Praxen [2]. MEDIAS 2 steht für "Mehr Diabetes Selbstmanagement für Typ 2“. Bei MEDIAS 2 BASIS handelt es sich um ein Schulungs- und Behandlungsprogramm für nicht insulinpflichtige Menschen mit Typ-2-Diabetes, ICT wurde speziell für Patienten mit einer intensivierten Insulintherapie entwickelt. Beide Programme sind für Disease Management Programme (DMP) zugelassen.

Das Forschungsinstitut der Diabetes-Akademie Mergentheim (FIDAM) und Lilly Diabetes sind zwei starke Partner, die eine langjährige, erfolgreiche Kooperation pflegen. Seit mittlerweile 15 Jahren arbeitet Lilly Diabetes mit dem FIDAM zusammen an der Entwicklung und Bereitstellung von Schulungs- und Behandlungsprogrammen: seit 1997 mit der Einführung von MEDIAS 2.

Weitere Informationen sind unter www.lilly-diabetes.de sowie www.medias2.de zu finden.

1. Kulzer B et al [Poster]. DDG, Stuttgart, 16.-19. Mai 2012; P123 2. FIDAM data on file

Quelle: Lilly 